\title{
Thrombospondin-targeting TAX2 peptide impairs tumor growth in preclinical mouse models of childhood neuroblastoma
}

\author{
Albin Jeanne ${ }^{1,2,3}$, Laurent Martiny ${ }^{1,2}$ and Stéphane Dedieu ${ }^{1,2}$
}

BACKGROUND: We have previously identified TAX2 peptide as an orthosteric antagonist for thrombospondin-1 (TSP-1) interaction with the cell-surface receptor CD47. TAX2 displays exciting antiangiogenic, antitumor, and antimetastatic properties in both allograft and xenograft models of melanoma as well as pancreatic carcinoma. Here, TAX2 therapeutic potential was investigated in two distinct preclinical mouse models of neuroblastoma.

METHODS: SK-N-BE(2) (MYCN-amplified) and SK-N-SH (MYCNnegative) human neuroblastoma cells have been implanted in outbred NMRI nude mice prior to systemic administrations of TAX2, and then tumor growth as well as intratumoral blood flow were longitudinally monitored. At study termination, subcutaneous xenografts were macroscopically and histopathologically examined.

RESULTS: In both models, TAX2 induced a significant inhibition of tumor burden in mice engrafted with large preestablished neuroblastoma tumors. Indeed, TAX2 administered at biologically relevant doses sharply alters xenograft vascularization as well as multiple features of tumor progression.

CONCLUSION: Altogether, our results present TAX2 peptide specifically targeting TSP-1:CD47 interaction as a new putative therapeutic approach for treating neuroblastoma, whether utilized alone or in combination with existing chemotherapy drugs.

N euroblastoma is the most common extracranial solid tumor in children under $5 \mathrm{y}$ old, indeed this highly vascular tumor of neural crest origin accounts for over $15 \%$ of pediatric cancer deaths (1). Neuroblastoma tumors are associated with extreme clinical heterogeneity in terms of disease progression and outcome as well as regarding response to treatment. Hence, progresses in neuroblastoma subtype classification have led to the development of various tailored therapeutic approaches (2). Among neuroblastoma most relevant drivers, amplification of oncogenic MYCN occurs in about $25 \%$ of cases and is associated with advance-staged disease (3). Nevertheless, a significant proportion of patients with no MYCN amplification also show poor prognosis (4). On the other hand, epigenetic alterations as well as a CpG island methylator phenotype (CIMP) are also associated with poor outcome (5) and contribute to $M Y C N$ overexpression (6). Notwithstanding the recent advances in disease genetic and epigenetic characterization of neuroblastoma (7), clinical outcome after intensive multimodal therapy remains poor for high-risk patients and additional prognostic markers and/or therapeutic targets are thus urgently warranted.

Access to blood vessels is a primary requirement of malignant neuroblastoma tumors in order to grow, invade, and form metastases (8), and a high vascular index strongly correlates with poor prognosis (9). Therefore, inhibiting tumor vascularization by using antiangiogenic drugs may provide a realistic therapeutic approach for the treatment of neuroblastoma. To date, clinical effectiveness of antiangiogenic treatment however remains unsatisfactory. In phase I clinical trials, the vascular endothelial growth factor (VEGF)-targeting monoclonal antibody bevacizumab have demonstrated significant but limited responses $(10,11)$. Among the explanations that could be put forward to such marginal improvement, one can criticize that most antiangiogenic strategies are aimed at targeting VEGF and/or its cognate tyrosine kinase receptors (VEGFR-1, VEGFR-2, and VEGFR-3). Indeed, inherent or acquired resistance may occur in patients, therefore leading to disease recurrence (12). In addition, a prolonged inhibition of VEGF in neuroblastoma xenografts has been shown to increase local hypoxia and to upregulate other angiogenic cytokines, therefore promoting cancer regrowth and metastasis (13). An alternative approach to face these limits and to improve tumor response might be to combine the use of bevacizumab with a backbone chemotherapy regimen. This is currently being pursued in phase IIb by extensively investigating the potential of adding bevacizumab to Temozolomide \pm Irinotecan as part of the BEACON-neuroblastoma trial (clinicaltrials.gov identifier NCT02308527). Yet, recent outstanding work pointed out the substantial contribution of VEGF-Ax, a novel antiangiogenic isoform of VEGF-A (14). By indiscriminately targeting both canonical proangiogenic

\footnotetext{
'Université de Reims Champagne-Ardenne, UFR Sciences Exactes et Naturelles, Reims, France; ${ }^{2}$ CNRS UMR 7369, Matrice Extracellulaire et Dynamique Cellulaire, MEDyC, Reims, France; ${ }^{3}$ SATT Nord, Lille, France. Correspondence: Stéphane Dedieu (stephane.dedieu@univ-reims.fr) 
and antiangiogenic VEGF isoforms, bevacizumab may inevitably lead to negative consequences in cancer treatment that are likely to contribute to its relative ineffectiveness (15). For all of the above-mentioned reasons, we are deeply convinced that original approaches and new molecular targets are needed in the purpose of efficiently inhibiting neuroblastoma tumor vascularization.

Thrombospondin-1 (TSP-1) is a well-known matricellular angiogenesis modulator that has been early identified as a putative marker of neuroblastoma differentiation (16). More recently, several studies suggested a key role for THBS1 (i.e., TSP-1 encoding gene) expression as the link between the angiogenic and epigenetic switches in neuroblastoma $(17,18)$. In previous work, we identified and characterized an innovative cyclic peptide, named TAX2, that selectively binds TSP-1 in order to antagonize its binding to CD47 membrane receptor $(19,20)$. TAX2 systemic administrations allowed significant antitumor and antimetastatic responses in several preclinical allograft and xenograft models of melanoma as well as pancreatic carcinoma, together with a potent disruption of tumor-associated vascular network. In the present study, we first provide a thorough analysis of THBS1 and CD47 expression analysis in both neuroblastoma clinical samples and cell lines based on publicly available data. Efficacy of the TSP-1:CD47 interaction-targeting TAX2 peptide was then tested in mice bearing large pre-established human neuroblastoma tumors. In two distinct models, i.e., the SK-N-BE(2) (MYCN-amplified) and SK-N-SH (no $M Y C N$ amplification) cell lines respectively established from 22-mo-old and 4-y-old patients with disseminated neuroblastoma (21), TAX2 achieved a marked inhibition in growth of s.c. tumors xenografted to outbred NMRI mice, while significantly decreasing intratumoral blood flow. These preclinical data suggest that thrombospondin-targeting TAX2 peptide may represent a valuable alternative approach for therapy in pediatric neuroblastoma.

\section{METHODS}

\section{Data Mining of Gene Expression Microarray Datasets}

TSP-1, CD47 and N-Myc encoding genes (i.e., THBS1, CD47, and $M Y C N$, respectively) were assessed for mRNA expression in Gene Expression Omnibus (GEO (22); and ArrayExpress (EMBL-EBI, Cambridgeshire, UK) databases as well as in the Cancer Cell Line Encyclopedia (23). Genevestigator v5 software (Nebion, Zurich, Switzerland) was used to retrieve and analyze data. Publicly available Versteeg gene expression dataset (24) was obtained from R2 microarray analysis and visualization platform (http://r2.amc.nl). Cut-off value for separating high and low THBS1 expression groups was determined by the online algorithm.

\section{Cell Lines}

The human neuroblastoma cell lines SK-N-BE(2) and SK-N-SH were obtained from the American Type Culture Collection (ATCC, Manassas, VA) and respectively maintained in Dulbecco's Modified Eagle Medium (DMEM)/nutrient mixture F12 and DMEM media (with L-glutamine) supplemented with $10 \%$ heat-inactivated fetal bovine serum. Cells were cultured in growth medium and split every $3-4 \mathrm{~d}$ by harvesting in trypsin.

\section{Formulations}

Preparation of the dosing solutions was on a daily basis at the administration day. TAX2 peptide (CEVSQLLKGDAC) acting as a specific antagonist for TSP-1:CD47 interaction (19) was synthesized and purified by Genecust (Dudelange, Luxembourg) and then controlled for composition and purity through electrospray ionization-mass spectrometry (ESI-MS) and high performance liquid chromatography (HPLC). Disulfide bridge cyclisation of the peptide was ensured by Raman spectroscopy. On the day of dosing, TAX2 was dissolved with injectable saline (Fresenius Kabi, Sèvres, France) to reach final concentrations of 3 to $10 \mathrm{mg} / \mathrm{ml}$ prior to the i.p. injection $(10 \mathrm{ml} / \mathrm{kg})$. Ready-to-use vehicle was used as a control $(10 \mathrm{ml} / \mathrm{kg})$. The alkylating agent cyclophosphamide (CTX) was purchased as a stock powder from Sigma-Aldrich (SaintQuentin Fallavier, France) and dissolved with injectable saline to reach a final concentration of $15 \mathrm{mg} / \mathrm{ml}(10 \mathrm{ml} / \mathrm{kg})$.

\section{Animal Care}

Eight-week-old female outbred NMRI (RjOrl:NMRI-Foxn $1^{n u} /$ Foxn $1^{n u}$ ) nude mice (average BW, $32 \pm 2 \mathrm{~g}$ ) were purchased from Janvier Labs (Saint-Berthevin, France). Animal handling was performed according to guidelines of the Federation of European Laboratory Animal Science Associations. The animals were fed a standard laboratory diet with water and food ad libitum and were kept under constant environmental conditions. This study was performed in compliance with "The French Animal Welfare Act" and following "The French Board for Animal Experiments". Experiments were conducted under approval of the French "Ministère de l'Enseignement Supérieur et de la Recherche" (ethics committee ${ }^{\circ} \mathrm{C} 2 \mathrm{EA}-75$ ) in compliance with the "Directive 2010/63/UE".

\section{Xenograft Models}

$5 \times 10^{6}$ SK-N-BE(2) or $1 \times 10^{7}$ SK-N-SH neuroblastoma cells in suspension within $100 \mu \mathrm{l}$ of PBS/Matrigel (1:1 mix) were s.c. inoculated into the right flank of NMRI nude mice. Tumor measurements (length $\mathrm{L}$, width $\mathrm{W}$ ) were performed twice a week using a digital caliper, and total tumor volume was calculated according to $\mathrm{V}=0.5 \times \mathrm{L} \times \mathrm{W}^{2}$. Systemic (i.p.) administrations of TAX2 (30 mg/kg to $100 \mathrm{mg} / \mathrm{kg} \mathrm{BW)}$ or injectable saline $(0.9 \% \mathrm{NaCl})$ were performed three days a week for $3 \mathrm{wk}$ starting when mean tumor volume reached $450-500 \mathrm{~mm}^{3}$ by group $(n=7)$. The reference treatment, i.e. cyclophosphamide (CTX) at its maximum tolerated dose (MTD; $150 \mathrm{mg} / \mathrm{kg} \mathrm{BW)} \mathrm{(25),} \mathrm{was} \mathrm{i.p.}$ administered once a week for $3 \mathrm{wk}$. Body weight (BW) was monitored twice a week while clinical signs and behavior pattern were daily checked until study termination. At study termination, mice were sacrificed and tumors were surgically extracted and fixed in $3.7 \%$ formalin for further morphologic studies.

\section{LASER Doppler Scanning}

At the time of group allocation (when tumor volume reached $450-500 \mathrm{~mm}^{3}$ ), four animals per group were randomly selected for LASER Doppler scanning (angiogenesis evaluation). The procedure was performed $1 \mathrm{~d}$ prior to the start of the treatment (baseline) and thereafter once a week until study termination. Blood flow within the s.c. tumor area was measured with a non-contact LASER Doppler. Normalized values were calculated in order to compare results between groups.

\section{Histology}

Paraffin-embedded s.c. xenografts of four animals per group were randomly subjected to histology analysis after routine trichrome staining. Hemalun-eosin-saffron-stained sections were analyzed qualitatively and semi-quantitatively for the following findings: morphologic type, percentage of necrosis, mitosis numeration (performed twice, independently, on 10 high power $\times 400$ fields). Analyses were performed by two veterinary pathologists who were blinded to the group's treatment during the whole procedure (macroscopic description after mid-sagittal section and histology analyses).

\section{Statistical Analyses}

Prism 5.0 software (GraphPad Software, La Jolla, CA) was used for all statistical analyses. Data are presented as mean \pm SEM. Significance was assessed using non-parametric Mann-Whitney $U$ test for unpaired samples. Multiple comparisons were performed using two-way ANOVA followed by post-hoc Dunnett's test. Logrank test was considered to compare the survival distributions of low $v s$. high THBS1-expressing groups. Two-sided $P$ values $<0.05\left(^{*}\right),<0.01\left(^{(*}\right)$ or $<0.001(\$)$ are indicated when statistical significance is reached. 


\section{Articles $\mid$ Jeanneet al.}

\section{RESULTS}

\section{THBS1 and CD47 Expression in Neuroblastoma Primary Tumors} and Cell Lines

Microarray gene expression profiling considering a large number of clinical samples highlighted that TSP-1-encoding gene (THBS1) is characterized by a very considerable heterogeneity in terms of expression in both neuroblastoma primary tumors and their normal counterparts, i.e., the adrenal gland cortex (Figure 1a). On the contrary, the ubiquitously expressed receptor CD47 appeared to be systematically highly expressed among these groups. Therefore, we then examined the relationship of THBS1 mRNA expression to overall survival in neuroblastoma patients. As shown in Figure 1b, high THBS1 mRNA levels were associated with poor outcome, whereas low THBS1 expression was associated with good prognosis among the Versteeg dataset consisting of a cohort of 88 neuroblastoma patients (24). Interestingly, analysis of mRNA expression levels in the Gene Expression Omnibus database suggests a relationship between reported MYCN gene amplification and low THBS1 expression (Figure 1c). Consistently, THBS1 mRNA inversely correlates with $M Y C N$ expression in the $M Y C N$ amplified SK-N-BE(2) and nonamplified SK-N-SH neuroblastoma cell lines (Figure 1d and Figure 1f), while they both display high CD47 mRNA expression (Figure 1e).

\section{TAX2 Peptide Inhibits Tumor Growth in the SK-N-BE(2) Xenograft Model}

To investigate whether targeting TSP-1:CD47 interaction could prevent neuroblastoma tumor growth, SK-N-BE(2) cells were subcutaneously xenotransplanted to outbred NMRI athymic nude mice prior to systemic administrations of TAX2 peptide. In this preclinical model, TAX2 i.p. delivery led to a $40 \%$ decrease in calculated tumor volume (Figure $2 \mathrm{a}$ ). Of note, no treatment-related toxicity was reported as neither adverse clinical signs nor body weight loss (Figure 2b) nor mortality/ morbidity were detected. Interestingly, tumor vascularization follow-up by LASER Doppler scanning highlighted a significant $40 \%$ decrease in measured blood flow under TAX2 treatment (Figure 2c). Analysis of individual responses (Figure 2d) revealed that most animals respond to TAX2 treatment, with four animals out of seven showing stable disease (SD) or partial response (PR). Noteworthy, one TAX2-treated mouse exhibited full disease regression (complete response, CR) on day 21 even though tumor volume exceeded $700 \mathrm{~mm}^{3}$ after $10 \mathrm{~d}$ of treatment. On the contrary, the magnitude of pre-established tumor burden was significantly higher in absence of TAX2 treatment (Figure 2e,f), with continuous tumor growth over time and no reported tumor regression in the control group (Figure 2g).

\section{TAX2 Treatment Sharply Modifies SK-N-BE(2) Tumors Morphological Pattern}

Consistent with caliper measurements performed throughout the study, the average of calculated tumor volume at necropsy was decreased by $40 \%$ in TAX2-treated group relatively to the control group (Figure 3a). TAX2 treatment also reduced tumor weight by about 30\% (Figure 3b). Macroscopic observation of tumors after mid-sagittal section confirmed that TAX2 delivery led to decreased size of s.c. SK-N-BE(2) xenografts which also exhibited a less dark color, probably indicating lower tumor vascularization and/or increased necrosis (Figure 3c). Morphologic description was further performed on Hemaluneosin-saffron-stained tumor sections. The histologic pattern of control animals (vehicle group) was neuroendocrine-like, i.e., cells arranged in solid nests separated by a thin and highly vascularized stroma (Figure 3d, top panel). Marked vascularization and hemorrhages explain the dark brown color observed macroscopically (Figure 3c, top panel). A sharp decrease in vascularization associated with a solid histologic pattern was observed in TAX2-treated group (Figure 3d, bottom panel), corresponding to the white to tan color observed macroscopically (Figure 3c, bottom panel). As shown in Figure 3e, necrotic scores were also higher under TAX2 treatment as compared to the vehicle group (see color-coded quantification bars). Finally, TAX2 also induced a significant $60 \%$ decrease in tumor mitotic index (Figure 3f). Taken together, this first round of data demonstrates that targeting TSP-1:CD47 interaction in the SK-N-BE(2) model affects multiple features of tumor xenografts, and suggests that TAX2 peptide may rise new expectancies for hampering neuroblastoma progression.

\section{TAX2 Dose-Dependently Inhibits Tumor Progression in the SK-N-SH Xenograft Model}

In order to extend the proof-of-concept of TAX2 efficacy, we then considered the SK-N-SH cell line (MYCN-negative status) to establish a second model of tumor xenografts, according to the same experimental design described above. In this additional study, increasing doses of TAX2 peptide have been tested and further compared to a standard of care treatment for high-risk neuroblastoma $(2,26)$. In this model, TAX2 inhibited tumor growth in a dose-dependent manner to reach a decrease of about $50 \%$ in s.c. xenograft volume at the highest dose (Figure $4 \mathrm{a}$ and Figure $4 \mathrm{~b}$ ). During the first $10 \mathrm{~d}$ of treatment, TAX2 peptide high dosing even yielded similar inhibition rates as those obtained with the chemotherapeutic agent cyclophosphamide (CTX) when used at its MTD (27). No difference between the vehicle group and TAX2-treated groups was observed regarding BW measurements, indicating that TAX2 treatment was well tolerated (Figure 4c). On the contrary, cyclophosphamide-treated group had a significantly decreased normalized BW that could be regarded as a treatment-related adverse effect. Indeed, TAX2 high dose and cyclophosphamide treatments reached similar benefits when looking at overall survival (Figure 4d).

\section{TAX2 Treatment Inhibits Angiogenesis in Pre-established SK-N- SH Tumor Xenografts}

While intratumoral blood flow measured in vehicle-treated animals increased by around 50\% during study period, TAX2 treatment significantly decreased (40\%) tumor blood supply at both low and high doses (Figure 5a). Consistently, histopathological analyses revealed that a solid pattern and a 
a

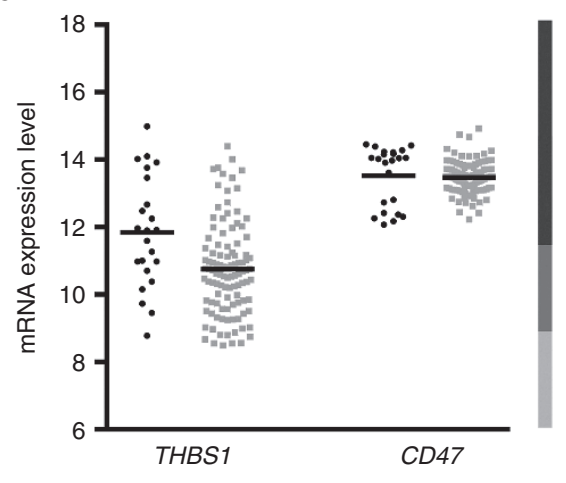

C

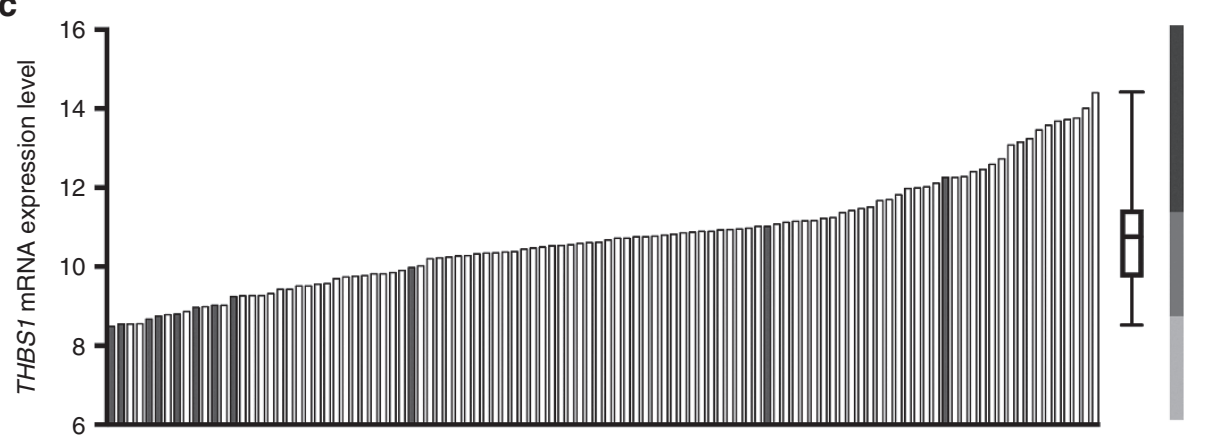

d

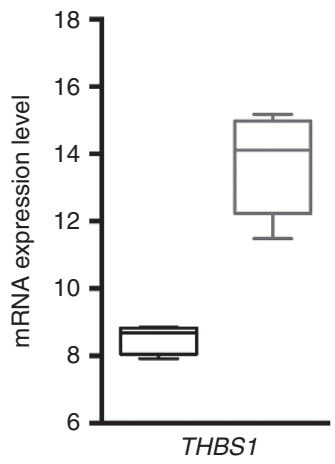

b

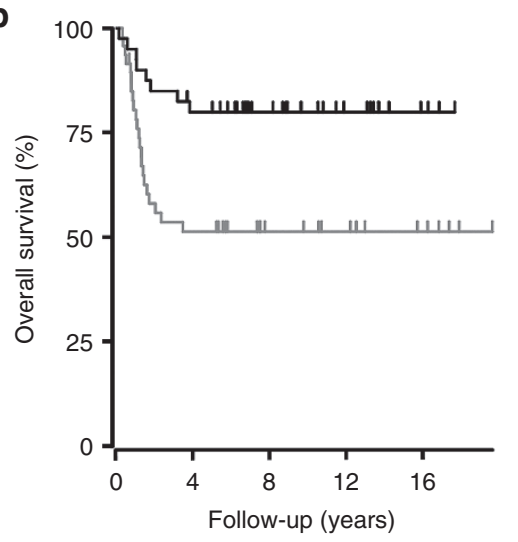

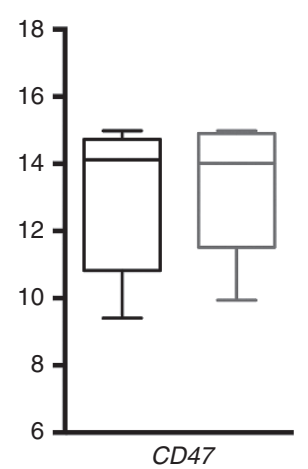

f

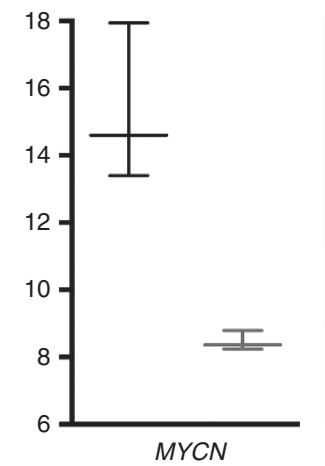

Figure 1. Expression analysis of TSP-1 and CD47-encoding genes in neuroblastoma. (a) Expression of THBS1 and CD47 mRNA in adrenal gland and neuroblastoma tumors. mRNA expression levels (log2; Affymetrix U133plus2.0 signal) were analyzed from microarray data resulting from public genomic profiles of normal adrenal gland cortex, i.e. neuroblastoma normal counterpart (black circles; GEO accession numbers GSE10927 and GSE19750, ArrayExpress E-MEXP-31), and neuroblastoma tumors (gray squares; GEO accession numbers GSE13136, GSE14880 and GSE16237). Lines correspond to mean, while a gray-scale bar indicates the typical range of low, medium (interquartile range) and high expression for this array type. (b) High THBS1 mRNA levels are significantly associated with poor prognosis in neuroblastoma patients. Kaplan-Meier survival curve was generated from R2 genomics analysis and visualization platform (http://r2.amc.nl) and shows the overall survival probability for high (gray line, $n=48$ ) vs. low (black line, $n=40)$ THBS1 mRNA expression in patients with neuroblastoma among the Versteeg dataset (GEO accession number GSE16476). Logrank test $P=0.0053$. (c) THBS1 gene expression inversely correlates with oncogenic MYCN in human neuroblastoma. Raw data from the Gene Expression Omnibus database are displayed on the histogram in which each patient/lesion case is represented by a bar that is coded to MYCN amplification status: white, no amplification reported; gray bar, MYCN gene amplification. Box-and-whiskers plot (line: median value, box: $25-75$ percentile, whiskers: $\mathrm{min} / \mathrm{max}$ values) stands for THBS1 gene expression along the whole dataset. (d-f) THBS1 (d), CD47 (e) and MYCN (f) mRNA expression levels (log2; Affymetrix U133plus2.0 signal) in SK-N-BE(2) (black) and SK-N-SH (gray) human neuroblastoma cell lines. Data were obtained from Broad-Novartis Cancer Cell Line Encyclopedia (GEO accession number GSE36133). Boxes show the median and interquartile range, while bottom and top bars of the whisker indicate the 10th and 90th percentiles, respectively.

marked decrease in vascularization are associated with TAX2 treatment (Figure 5b). In addition, decreased mitotic scores and increased number of apoptotic bodies were found in TAX2-treated groups, in a similar extent than in the positive control cyclophosphamide-treated group when considering TAX2 peptide high dose (data not shown). Finally, measurements of excised tumors highlighted a 40\% decrease in both tumor volume and tumor weight under TAX2 treatment (Figure 5c and Figure 5d). Altogether, these data shed light on exciting anti-angiogenic and anti-tumor properties of TAX2 peptide in two distinct preclinical models of neuroblastoma. 
a

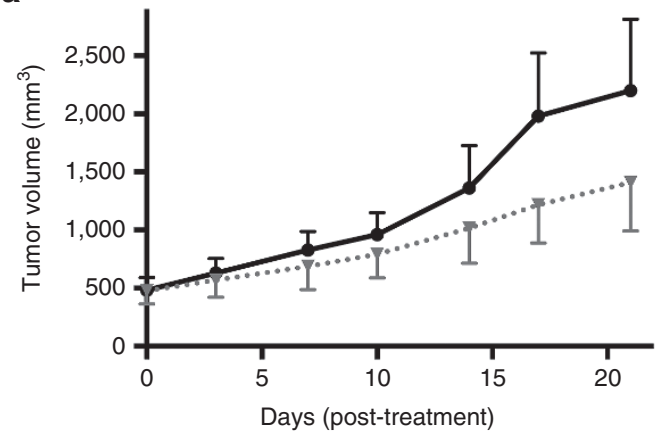

C

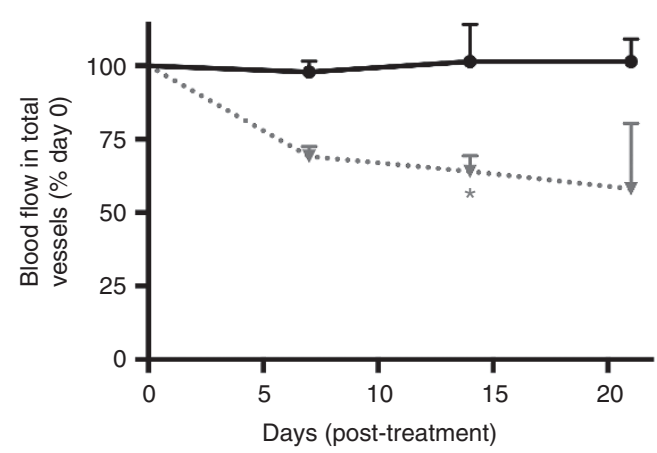

b

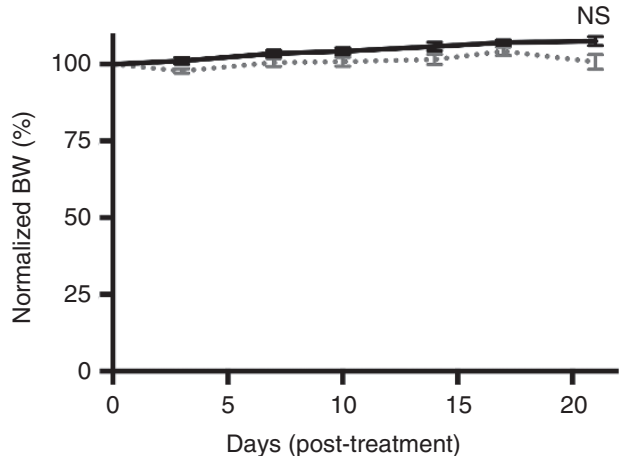

d

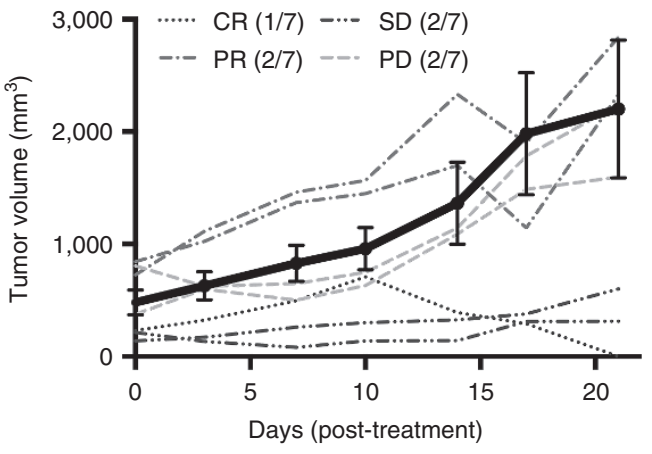

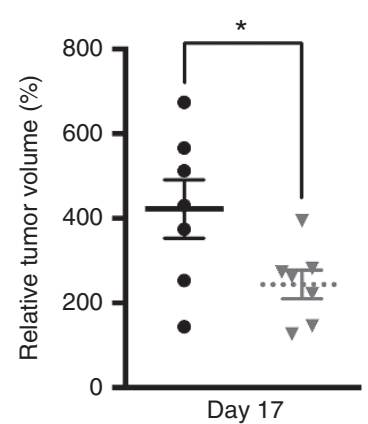

$\mathbf{f}$

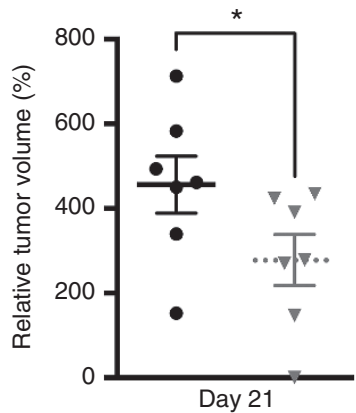

g

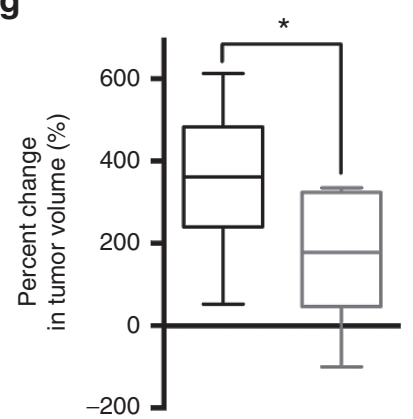

Figure 2. Therapeutic evaluation of TAX2 peptide in the SK-N-BE(2) xenograft model. $5 \times 10^{6} \mathrm{SK}-\mathrm{N}-\mathrm{BE}(2)$ neuroblastoma cells were s.c. implanted in NMRI nude mice and then tumor xenografts were allowed to grow until $500 \mathrm{~mm}^{3}$. After randomization, vehicle $(0.9 \% \mathrm{NaCl})$ or TAX2 peptide (100 mg/kg BW) were i.p. injected three times a week for $3 \mathrm{wk}$ ( $n=7$ per group). LASER Doppler monitoring was performed once a week along the whole procedure. Mice were regularly checked for BW, tumor volume and clinical signs. (a) Averages of calculated SK-N-BE(2) tumor xenograft volume in $\mathrm{mm}^{3}$ for control (solid black line and circles) or TAX2-treated (black dotted line and triangles) groups (mean $\pm \mathrm{SEM}, n=7$ ). (b) Evolution of normalized BW, expressed as a percentage of day 0 for control (solid black line and circles) or TAX2-treated (black dotted line and triangles) mice (mean \pm SEM, $n=7$, Mann-Whitney U-test; NS, not significant). (c) Angiogenesis evaluation in SK-N-BE(2) xenografts. Intratumoral blood flow was measured with a non-contact LASER Doppler at baseline (before treatment) and then once a week during 3 wk. Histogram shows averages of normalized blood flow as a percentage of day 0 for control (solid black line and circles) or TAX2-treated (black dotted line and triangles) mice (mean $\pm \mathrm{SEM}, n=4$, Mann-Whitney $U$-test, $\left.{ }^{*} P<0.05\right)$. (d) Individual tumor volume responses to TAX2 treatment. Calculated tumor volume of each TAX2-treated tumor is plotted as a function of time (days post-treatment), while tumor volume evolution in the control group $(0.9 \% \mathrm{NaCl})$ is presented as mean $\pm \mathrm{SEM}$ (black bold line). TAX2-treated subgroups are represented according to tumor responses as follows: CR, complete response (i.e., all detectable tumor has disappeared, dotted); SD, stable disease (includes either a small amount of growth or a small amount of shrinkage, dash/dot-dot); PR, partial response (i.e., transient shrinkage with evidence of some residual disease, dash/dot) and PD, progressive disease (no response to treatment, dashed). (e-f) Relative SK-N-BE(2) tumor xenograft volumes on days 17 (e) and 21 (f) for control (black circles) and TAX2-treated (gray triangles) mice. Individual calculated tumor volumes on each time point are presented relatively to corresponding tumor volumes on day $0\left(n=7\right.$, Mann-Whitney $U$-test, $\left.{ }^{*} P<0.05\right)$. (g) Box-and-whiskers plot (line: median value, box: $25-75$ percentile, whiskers: $\mathrm{min} /$ max values) represents the percent change in tumor volume on day 21 for control (black) and TAX2-treated (gray) animals ( $n=7$, Mann-Whitney $U$-test, $\left.{ }^{*} P<0.05\right)$

\section{DISCUSSION}

In this study, we used two heterotopic xenograft mouse models of human neuroblastoma in order to assess the therapeutic efficacy of the thrombospondin-targeting TAX2 peptide. In both the SK-N-BE(2) and SK-N-SH tumor xenotransplantation models, respectively corresponding to $M Y C N$-amplified and non-amplified neuroblastoma cells, TAX2 peptide targeting TSP-1:CD47 interaction inhibited s.c. tumor growth by about two-fold (Figure 2a and Figure 4a). Concomitantly, tumor vascularization was significantly altered under TAX2 treatment 
a

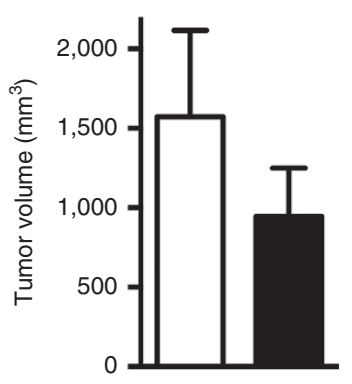

b

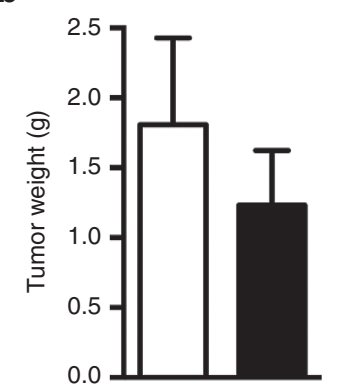

C

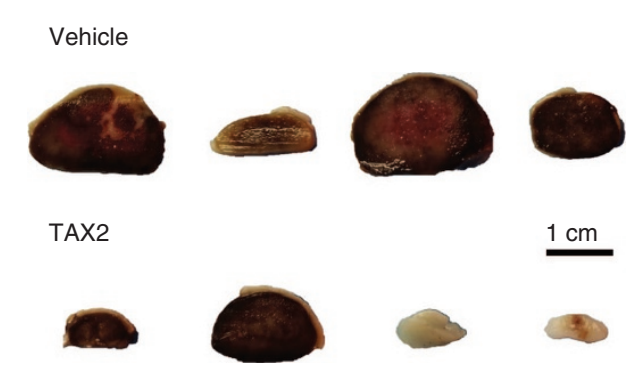

d
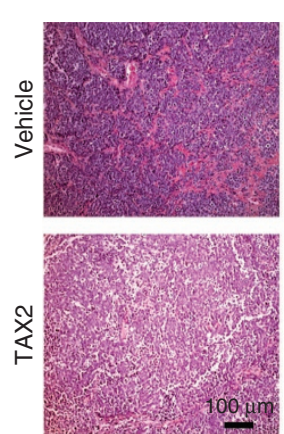

e

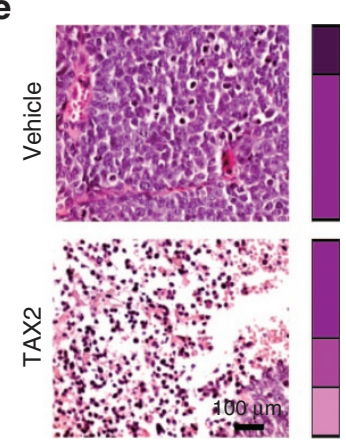

$\mathbf{f}$

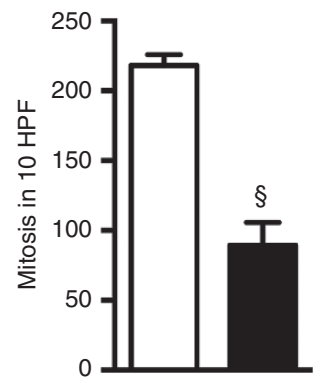

Figure 3. Macroscopic and histologic analyses of isolated SK-N-BE(2) tumors.

(a) Averages of s.c. xenograft volume in $\mathrm{mm}^{3}$ after tumor excision from control (black pattern) and TAX2-treated (gray checkerboard pattern) mice (mean \pm SEM, $n=7$ per group). (b) Averages of tumor weight in $\mathrm{g}$ at study termination for control (black pattern) and TAX2-treated (gray checkerboard pattern) mice (mean $\pm \mathrm{SEM}, n=7$ ). (c) Representative macroscopic views of SK-N-BE(2) neuroblastoma subcutaneous xenografts after mid-sagittal section. A less dark color in the TAX2-treated group indicates less vascularized tumors and/or increased necrosis. (d) Representative microscopic $\times 100$ view of hemalun-eosin-saffron (HES)-stained SK-N-BE(2) tumor xenograft sections. Vehicle ( $0.9 \% \mathrm{NaCl}$ ) group: neuroendocrine-like pattern, marked vascularization; TAX2-treated group: solid pattern with low vascularization. (e) Microscopic $\times 400$ view of HES-stained sections allowing necrosis visualization within tumors from TAX2-treated group. Color bar displays repartition of necrotic scores among tumors from each group, according to the following classification (from dark to light purple): isolated apoptotic bodies, small necrotic foci $<25 \%$, necrotic areas 25 to $50 \%$, necrotic areas 51 to $75 \%$. (f) Quantification of the number of mitosis on 10 high power $\times 400$ fields within viable area of tumors from control (black pattern) and TAX2-treated (gray checkerboard pattern) mice (performed twice, independently, $n=4$, Mann-Whitney $U$-test, $\S P<0.001$ ).

(Figure 2c and Figure 5a). Interestingly, TAX2 administration at biologically relevant doses $(10 \mathrm{mg} / \mathrm{kg} /$ day $)$ was found to be safe as it did not display any toxicity in these models, while cyclophosphamide used as reference treatment significantly decreased mice BW (Figure 4c) and led to lethal adverse event for one animal out of six (Figure 4d).

TAX2 anti-cancer effects are mainly attributed to its robust antiangiogenic properties. We previously demonstrated that such angiostatic effect is the consequence of a potentiation of CD36 engagement. Indeed, by preventing CD47 ligation, TAX2 peptide increases TSP- 1 bioavailability and consequently CD36 engagement (19). Our present results are therefore consistent with previous work showing that a peptide derived from the CD36-binding sequence of TSP-1 also inhibits neuroblastoma xenograft growth by hampering tumor vascularization (28). Nevertheless, it seems obvious that TAX2 effects on neuroblastoma tumor growth may not only be dependent on angiogenesis. LASER Doppler measurements of intratumoral blood flow in the SK-N-SH model revealed that a maximal $40 \%$ inhibition rate is reached at a $30 \mathrm{mg} / \mathrm{kg}$ TAX2 dosing (Figure 5a). However, a dose-response effect is observed up to $100 \mathrm{mg} / \mathrm{kg}$ when considering tumor growth inhibition (Figure 4a), therefore strongly suggesting that other mechanisms may be involved. Indeed, CD47 activation under TSP-1 ligation is known to directly influence cancer cells by regulating a wide range of processes such as proliferation, survival, apoptosis and acquisition of an invasive phenotype $(29,30)$. By concomitantly activating CD36 together with a disruption of TSP-1/ CD47 signaling, TAX2 is likely to combine the positive effects of targeting stromal cells that drive tumor angiogenesis as well as those resulting from a direct impact on cancer cell behavior. Indeed TAX2 treatment increased necrotic areas within SK-N-BE(2) xenografts (Figure 3e) along with a significant decrease in tumor cell mitotic index (Figure 3f). Similarly, decreased mitosis and numerous apoptotic bodies were observed following TAX2 administration in the SK-N-SH model (data not shown). This is consistent with previous work reporting that CD47 ligation by TSP-1 protects thyroid carcinoma cells from apoptosis (31) and promotes proliferation of astrocytoma cells (32). In order to provide a better characterization of TAX2-mediated effects, further in vitro work as well as immunohistochemistry analyses of tumors will be aimed at deciphering the actual contribution of angiogenesis, proliferation and apoptosis in TAX2 anticancer properties. Taken together, information resulting from longitudinal blood flow measurements as well as histopathological analyses of isolated 


\section{Articles $\mid$ Jeanneet al.}

a

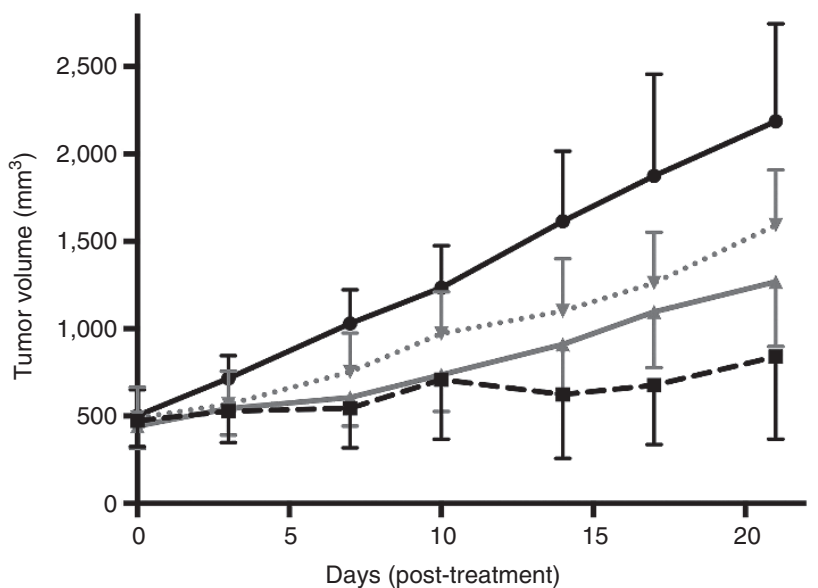

b

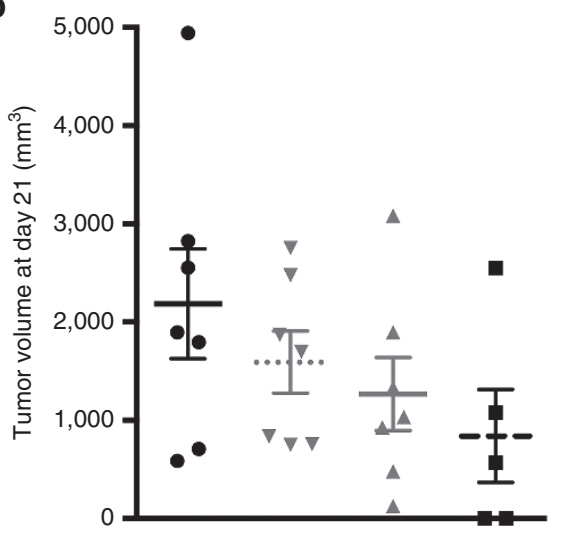

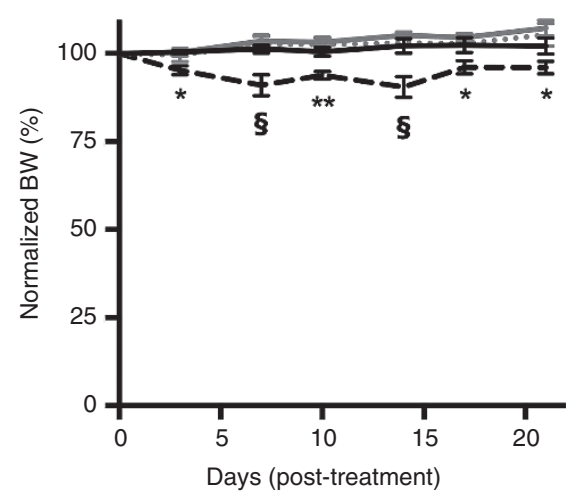

d

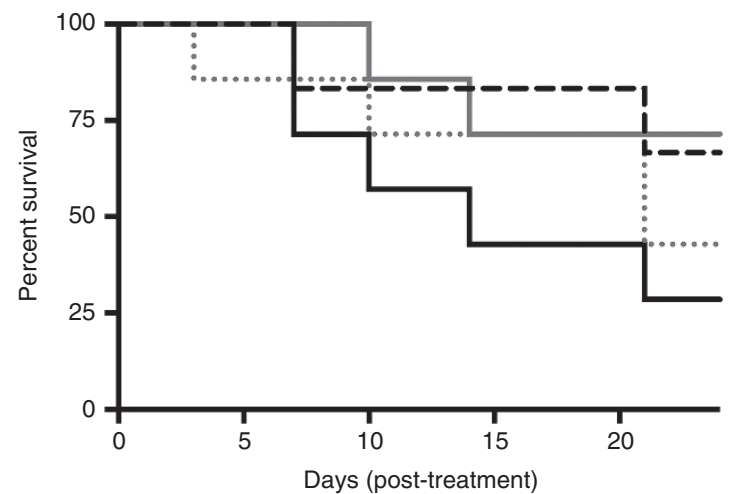

Figure 4. Dose-response and comparative study of TAX2 treatment in the SK-N-SH model. $1 \times 10^{7}$ SK-N-SH human neuroblastoma cells were s.c. implanted in NMRI nude mice (RjOrl:NMRI-Foxn $1^{n u} /$ Foxn $1^{n u}$ ) and then tumor xenografts were allowed to grow until $500 \mathrm{~mm}^{3}$. After randomization, vehicle $(0.9 \% \mathrm{NaCl})$, TAX2 peptide ( $30 \mathrm{mg} / \mathrm{kg}$ to $100 \mathrm{mg} / \mathrm{kg} \mathrm{BW}$ ) or cyclophosphamide (CTX, $150 \mathrm{mg} / \mathrm{kg} \mathrm{BW;} \mathrm{i.e.,} \mathrm{maximum} \mathrm{tolerated} \mathrm{dose)} \mathrm{were} \mathrm{i.p.} \mathrm{injected}$ 3 times a week (vehicle and TAX2 groups) or once a week (CTX group) for $3 \mathrm{wk}$ ( $n=6-7$ per group). (a) Averages of calculated tumor volume in mm ${ }^{3}$ for control (black solid line and circles), TAX2 low dose (gray dotted line and triangles) and high dose (gray solid line and triangles) as well as CTX-treated (black dashed line and squares) groups (mean \pm SEM, $n=6-7$ per group). (b) Individual calculated tumor volumes at day 21 for control (black circles), TAX2 low dose (gray downward-pointing triangles) and high dose (gray upward-pointing triangles) as well as CTX-treated (black squares) groups (mean \pm SEM). (c) Evolution of normalized body weights for control (black solid line), TAX2 low dose (gray dotted line) and high dose (gray solid line) as well as CTX-treated (black dashed line) mice, expressed as a percentage of day 0 (mean $\pm \mathrm{SEM}, n=6-7,{ }^{*} P<0.05,{ }^{* *} P<0.01, \S P<0.001$ compared to vehicle group). (d) Kaplan-Meier survival curves after experimental treatments of control (black solid line), TAX2 low dose (gray dotted line) and high dose (gray solid line) as well as CTX-treated (black dashed line) animals. Survival analysis was based on follow-up until tumor volumes exceeded 1,500 $\mathrm{mm}^{3}$ or euthanasia, which was conducted when mice were moribund.

human tumor xenografts nonetheless confirmed that through its original and differentiated molecular mechanism of action (19), TAX2 affects tumor vascularization as well as multiple parameters of neuroblastoma tumor progression.

Analysis of SK-N-BE(2) xenograft individual tumor responses to TAX2 treatment highlighted the relative heterogeneity of therapy effectiveness in this model, ranging from tumor total regression to nonresponsive animals (Figure 2d). Besides, results from gene expression analysis highlighted that MYCN amplification in the SK-N-BE(2) cell line correlates with low TSP-1 expression whereas high THBS1 mRNA levels were found in nonamplified SK-N-SH cells (Figure 1d,f). THBS1 mRNA levels extracted from publicly available databases are consistent with previous work highlighting TSP-1 protein expression by SK-N-SH cells whereas TSP-1 was neither detected in SK-N-BE(2) conditioned medium nor by immunochemistry (18). As both neuroblastoma cellular models strongly express CD47 receptor (Figure 1e), one should consider that TAX2 antitumor effects in the SK-N-BE(2) xenograft model mainly depend on TSP-1 enrichment within tumor stroma, especially arising from endothelial cells TSP-1 production (33). Indeed, bioavailable TSP-1 concentrations are likely to vary from one animal to another, particularly as outbred mice were considered in our efficacy studies in the purpose of better reflecting diversity existing within a natural population $(34,35)$. Accordingly, mining of multiple gene expression microarray data highlighted that THBS1 gene expression exhibits much heterogeneity among 106 clinical cases of neuroblastoma (Figure 1c). Data arising both from cell lines (Figure 1d,f) as well as neuroblastoma clinical samples (Figure 1c) suggested an inverse relationship between MYCN gene amplification and THBS1 expression. This should 
a

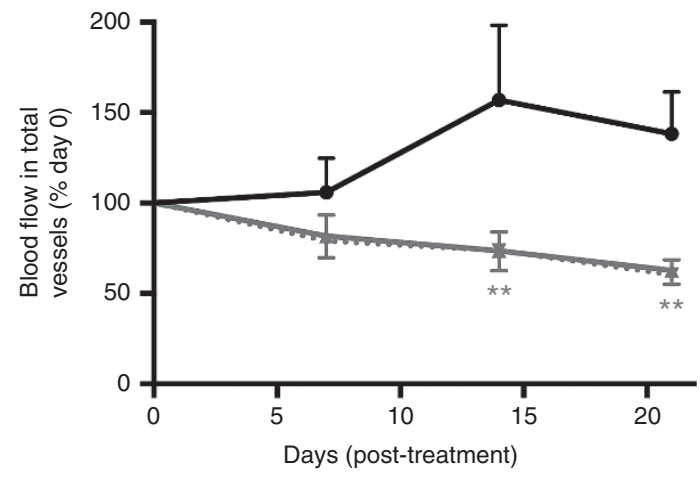

b
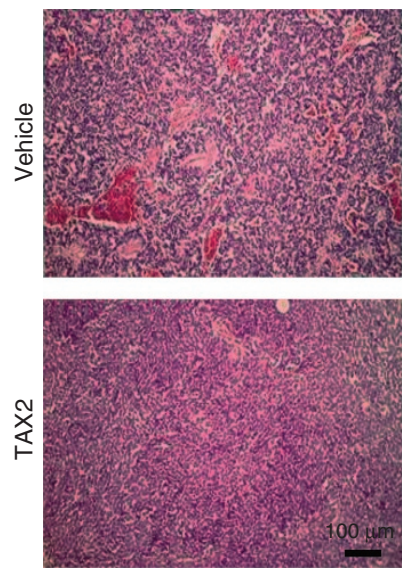

C

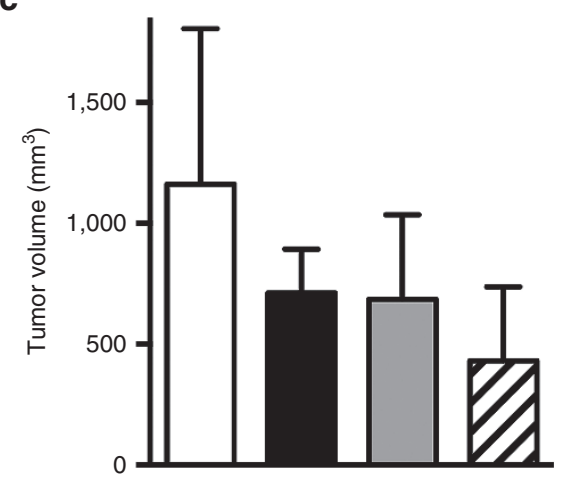

d

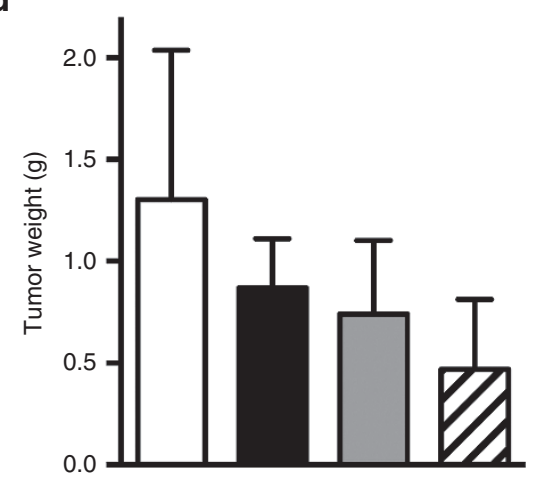

Figure 5. SK-N-SH tumors in vivo angiogenesis and ex vivo morphological pattern assessment. (a) In vivo intratumoral blood flow (total vessels) was measured with a non-contact LASER Doppler at baseline (before treatment) and then once a week during 3 wk. Graph shows averages of normalized blood flow in tumors as a percentage of day 0 for control (black solid line and circles), TAX2 low dose (gray dotted line and triangles) and high dose (gray solid line and triangles) groups (mean $\pm \mathrm{SEM}, n=4$, Mann-Whitney U-test, $* * P<0.01$ ). (b) Representative microscopic $\times 100$ view of HES-stained SK-N-SH tumor xenografts sections. Vehicle $(0.9 \% \mathrm{NaCl})$ group: neuroendocrine-like pattern, marked vascularization; TAX2-treated group (100 mg/kg BW): solid pattern with low vascularization (pattern associated with the treatment). (c) Averages of s.c. xenograft volume in $\mathrm{mm}^{3}$ after tumor excision from control (black pattern), TAX2 low dose (gray checkerboard pattern) and high dose (gray stripes) as well as CTX-treated (white pattern) mice (mean \pm SEM, $n=6-7$ per treatment-related group). (d) Averages of tumor weight in $\mathrm{g}$ at study termination for control (black pattern), TAX2 low dose (gray checkerboard pattern) and high dose (gray stripes) as well as CTX-treated (white pattern) groups (mean $\pm \mathrm{SEM}, n=6-7$ ).

be regarded in light of previous research that widely documented an interrelation between TSP-1/CD47 signaling and other member of oncogenic Myc transcription factors family, i.e., c-Myc (36,37). In neuroblastoma, THBS1 gene expression has been established as a putative link between the angiogenic and epigenetic switches (17). Indeed, a few strategies based on the use of demethylating agents were developed with the purpose of restoring THBS1 expression $(6,18)$. Nevertheless, full-length TSP-1 should rather be regarded as a double-edged sword within a tumor microenvironment, and THBS1 gene expression enhancement may led to inevitable adverse effects that are closely related to the pleiotropic nature of matricellular proteins and their ligands, which regulate tumor growth both positively and negatively (30). On the other hand, phase I clinical trials considering humanized anti-CD47 monoclonal antibodies directed against various solid tumors are currently underway (clinicaltrials.gov identifiers NCT 02216409, NCT02447354, NCT02488811, and NCT02367196). These are mainly aimed at blocking the antiphagocytic pathway that results from $\mathrm{CD} 47$ interaction with signal regulatory protein alpha $\operatorname{SIRP} \alpha(30,38)$. Given the ubiquitous expression of CD47, such antibodies may nevertheless promote severe side effects such as excessive elimination of erythrocytes. Indeed, CD47 antibody blockade is likely to exacerbate some clinical features in neuroblastoma patients such as severe anemia following bone marrow infiltration (39). Hence, TAX2 peptide is to date the first molecule that selectively disrupt TSP-1/CD47 signaling by targeting TSP-1 at the CD47 binding site, therefore allowing a fine regulation of the balance between TSP- 1 proand antitumor properties in neuroblastoma. Here, we provide a proof-of-concept for preclinical therapeutic efficacy of this innovative peptide agent in neuroblastoma. Further ADME and pharmacokinetics/pharmacodynamics studies as well as determination of TAX2 safety profile (repeated dosing, MTD determination) will help improving its translational potential toward clinical phases.

In our opinion, future research on neuroblastoma therapy should comprehensively integrate data generated from expression profiling as well as epigenetic and proteomic analyses in order to propose optimized personalized medicine. In this 
regard, TAX2 peptide provides new expectancies for antivascular therapy that may be part of a multiple target approach aiming at improving therapeutic responses and overcoming resistance.

\section{STATEMENT OF FINANCIAL SUPPORT}

The authors acknowledge support from SATT Nord and Centre National de la Recherche Scientifique (CNRS).

Disclosure: The authors declare no conflict of interest.

\section{REFERENCES}

1. Smith MA, Seibel NL, Altekruse SF, et al. Outcomes for children and adolescents with cancer: challenges for the twenty-first century. J Clin Oncol 2010;28:2625-34.

2. PintoNR,Applebaum MA, VolchenboumSL, etal.Advancesin riskclassification and treatment strategies for neuroblastoma. J Clin Oncol 2015;33:3008-17.

3. Brodeur GM, Seeger RC. Gene amplification in human neuroblastomas: basic mechanisms and clinical implications. Cancer Genet Cytogenet 1986;19:101-11.

4. Brodeur GM, Hogarty MD, Mosse YP, Maris JM. Neuroblastoma. In: Pizzo PA, Poplack DG, eds. Principles and Practice of Pediatric Oncology. Philadelphia, PA: Lippincott Williams \& Wilkins; 2011. pp. 886-922.

5. Abe M, Ohira M, Kaneda A, et al. CpG island methylator phenotype is a strong determinant of poor prognosis in neuroblastomas. Cancer Res 2005;65:828-34.

6. Gu S, Tian Y, Chlenski A, et al. Valproic acid shows a potent antitumor effect with alteration of DNA methylation in neuroblastoma. Anticancer Drugs 2012;23:1054-66.

7. Bagatell R, Cohn SL. Genetic discoveries and treatment advances in neuroblastoma. Curr Opin Pediatr 2016;28:19-25.

8. Roy Choudhury S, Karmakar S, Banik NL, Ray SK. Targeting angiogenesis for controlling neuroblastoma. J Oncol 2012;2012:782020.

9. Eggert A, Ikegaki N, Kwiatkowski J, Zhao H, Brodeur GM, Himelstein BP. High-level expression of angiogenic factors is associated with advanced tumor stage in human neuroblastomas. Clin Cancer Res 2000;6:1900-8.

10. Glade Bender JL, Adamson PC, Reid JM, et al.; Children's Oncology Group Study. Phase I trial and pharmacokinetic study of bevacizumab in pediatric patients with refractory solid tumors: a Children's Oncology Group Study. J Clin Oncol 2008;26:399-405.

11. Benesch M, Windelberg M, Sauseng W, et al. Compassionate use of bevacizumab (Avastin) in children and young adults with refractory or recurrent solid tumors. Ann Oncol 2008;19:807-13.

12. Okamoto S, Nitta M, Maruyama T, et al. Bevacizumab changes vascular structure and modulates the expression of angiogenic factors in recurrent malignant gliomas. Brain Tumor Pathol 2016;33:129-36.

13. Hartwich J, Orr WS, Ng CY, Spence Y, Morton C, Davidoff AM. HIF-1 $\alpha$ activation mediates resistance to anti-angiogenic therapy in neuroblastoma xenografts. J Pediatr Surg 2013;48:39-46.

14. Eswarappa SM, Potdar AA, Koch WJ, et al. Programmed translational readthrough generates antiangiogenic VEGF-Ax. Cell 2014;157:1605-18.

15. Eswarappa SM, Fox PL. Antiangiogenic VEGF-Ax: A New Participant in Tumor Angiogenesis. Cancer Res 2015;75:2765-9.

16. Castle VP, Ou X, O'Shea S, Dixit VM. Induction of thrombospondin 1 by retinoic acid is important during differentiation of neuroblastoma cells. J Clin Invest 1992;90:1857-63.

17. Lau DT, Hesson LB, Norris MD, Marshall GM, Haber M, Ashton LJ. Prognostic significance of promoter DNA methylation in patients with childhood neuroblastoma. Clin Cancer Res 2012;18:5690-700.

18. Yang QW, Liu S, Tian Y, et al. Methylation-associated silencing of the thrombospondin-1 gene in human neuroblastoma. Cancer Res 2003;63:6299-310.
19. Jeanne A, Sick E, Devy J, et al. Identification of TAX2 peptide as a new unpredicted anti-cancer agent. Oncotarget 2015;6: 17981-8000.

20. Jeanne A, Boulagnon-Rombi C, Devy J, et al. Matricellular TSP-1 as a target of interest for impeding melanoma spreading: towards a therapeutic use for TAX2 peptide. Clin Exp Metastasis 2016;33:637-49.

21. Barnes EN, Biedler JL, Spengler BA, Lyser KM. The fine structure of continuous human neuroblastoma lines SK-N-SH, SK-N-BE(2), and SK-NMC. In Vitro 1981;17:619-31.

22. Barrett T, Suzek TO, Troup DB, et al. NCBI GEO: mining millions of expression profiles-database and tools. Nucleic Acids Res 2005;33(Database issue):D562-6.

23. Barretina J, Caponigro G, Stransky N, et al. The Cancer Cell Line Encyclopedia enables predictive modelling of anticancer drug sensitivity. Nature 2012;483:603-7.

24. Molenaar JJ, Koster J, Zwijnenburg DA, et al. Sequencing of neuroblastoma identifies chromothripsis and defects in neuritogenesis genes. Nature 2012;483:589-93.

25. Emmenegger U, Francia G, Chow A, et al. Tumors that acquire resistance to low-dose metronomic cyclophosphamide retain sensitivity to maximum tolerated dose cyclophosphamide. Neoplasia 2011;13:40-8.

26. Emmenegger U, Man S, Shaked Y, et al. A comparative analysis of lowdose metronomic cyclophosphamide reveals absent or low-grade toxicity on tissues highly sensitive to the toxic effects of maximum tolerated dose regimens. Cancer Res 2004;64:3994-4000.

27. Shusterman S, Maris JM. Prospects for therapeutic inhibition of neuroblastoma angiogenesis. Cancer Lett 2005;228:171-9.

28. Yang Q, Tian Y, Liu S, et al. Thrombospondin-1 peptide ABT-510 combined with valproic acid is an effective antiangiogenesis strategy in neuroblastoma. Cancer Res 2007;67:1716-24.

29. Sick E, Jeanne A, Schneider C, Dedieu S, Takeda K, Martiny L. CD47 update: a multifaceted actor in the tumour microenvironment of potential therapeutic interest. Br J Pharmacol 2012;167:1415-30.

30. Jeanne A, Schneider C, Martiny L, Dedieu S. Original insights on thrombospondin-1-related antireceptor strategies in cancer. Front Pharmacol 2015;6:252.

31. Rath GM, Schneider C, Dedieu S, et al. Thrombospondin-1 C-terminalderived peptide protects thyroid cells from ceramide-induced apoptosis through the adenylyl cyclase pathway. Int J Biochem Cell Biol 2006;38:2219-28.

32. Sick E, Boukhari A, Deramaudt T, et al. Activation of CD47 receptors causes proliferation of human astrocytoma but not normal astrocytes via an Akt-dependent pathway. Glia 2011;59:308-19.

33. Brown LF, Guidi AJ, Schnitt SJ, et al. Vascular stroma formation in carcinoma in situ, invasive carcinoma, and metastatic carcinoma of the breast. Clin Cancer Res 1999;5:1041-56.

34. Chia R, Achilli F, Festing MF, Fisher EM. The origins and uses of mouse outbred stocks. Nat Genet 2005;37:1181-6.

35. Zeineldin M, Jensen D, Paranjape SR, et al. Human cancer xenografts in outbred nude mice can be confounded by polymorphisms in a modifier of tumorigenesis. Genetics 2014;197:1365-76.

36. Kaur S, Soto-Pantoja DR, Stein EV, et al. Thrombospondin-1 Signaling through CD47 Inhibits Self-renewal by Regulating c-Myc and Other Stem Cell Transcription Factors. Sci Rep 2013;3:1673.

37. Kaur S, Roberts DD. Divergent modulation of normal and neoplastic stem cells by thrombospondin-1 and CD47 signaling. Int J Biochem Cell Biol 2016;81(Pt A):184-194.

38. Chao MP, Weissman IL, Majeti R. The CD47-SIRP $\alpha$ pathway in cancer immune evasion and potential therapeutic implications. Curr Opin Immunol 2012;24:225-32.

39. Marino S, Puglisi F, Magro G, et al. Neuroblastoma: diagnostic and clinical aspects. Journal of Pediatric Biochemistry 2016;05:131-8. 